\title{
Editorial
}

\section{What's next ?}

Yvon Roche ${ }^{1,2,3,4, *}$

${ }^{1}$ Department of Oral Surgery, UFR d'Odontologie Garancière, Université de Paris, Paris, France

2 Service d'Odontologie, Hôpital Rothschild, AP-HP Sorbonne Université, Paris, France

${ }^{3}$ Ex-Clinical Fellow in Oral and Maxillofacial Surgery, University of California at Los Angeles, USA

${ }^{4}$ Ex-senior Lecturer in Oral and Maxillofacial Surgery, Sydney University Australia, Australia

(Received: 6 January 2021, accepted: 11 January 2021)

This year, the French dental specialty of Oral Surgery (OS) will be 10 years old. Happy birthday!!! In fact, 0 S and Orthodontics are the only dental specialties recognized by the European Union. The residency has a 4-year curriculum which is common to both dental and medical students. Today, 215 practitioners with a DDS degree are qualified- and 129 of them limit their practice to $0 \mathrm{~S}$. It is primarily an office-based practice, similar to the practice of many oral surgeons in the United States and in many other European countries. Positioned at the interface between dentistry and medicine, $O S$ requires training and competence in both disciplines.

Oral and Maxillofacial Surgery (OMFS) and Stomatology have long been practiced in France as medical specialties. OMFS is primarily hospital-based. The need for focused dental training has been discussed, but not accomplished. Cooperation between the two specialties would be highly desirable in the future, and would greatly benefit our patients.

Since the management of oral and maxillofacial diseases is of interest to both dentists and physicians, the following question arises: What is the best way to acquire the dental, medical, and surgical training needed to deal with these diseases? Must the training be degree-based, or can it be streamlined? In countries around the world, four basic pathways have evolved: training requiring a dental degree; training requiring a medical degree; training requiring both degrees; and training that includes both dental and medical education, but is not degree-based.

\section{Countries that require a dental degree}

OMFS training is open to dental graduates in many parts of the world. Some of these countries also offer dual degree programs (although the medical degree is not mandatory in

\footnotetext{
*Correspondence: yvon.roche@u-paris.fr
}

those countries). Appropriate medical and surgical training is an integral part of these programs. The countries include the United States, Canada, Russia, Japan, India, Pakistan, Bangladesh, Malaysia, Sri Lanka, and Hong Kong. Programs in Latin America, the Middle East, and Africa are also open to dental graduates. In Europe, Nordic countries also have programs open to dental graduates.

\section{Countries that require a medical degree}

OMFS training programs are run by medical faculties in France, Italy, Spain, and Portugal. In contrast to other European countries, there may be little or no formal dental training. These programs are separate from OS programs.

\section{Countries that require both dental and medical degrees}

Most of the countries in the European Union, the UK, Australia, and New Zealand require dual qualification for training in OMFS.

\section{Country that requires both dental and medical training, but no degree}

In China, training in both dentistry and OMFS is run by the schools of stomatology. There are 5-year, 7-year, and 8-year courses, leading to a Bachelor's degree, Master's degree and Doctorate in Stomatology. There are no formal OMFS curricula, but graduate students receive residency training at the teaching hospitals.

For centuries, dentistry and OS were practiced in France by itinerant tradesmen, who had little training and no regulation. Some physicians and surgeons, such as Pierre Fauchard and 
Ambroise Paré, also became interested in oral and maxillofacial diseases. For many years some physicians practiced OS. Over the world, there have beendental schoolsforless than 200 years. As dental training developed, OS became a dental practice and then a dentalspecialty. Then came the horrendous maxillofacial injuries encountered in the World Wars of the 20th century. Unprecedented, novel treatments were carried out by a host of skillful dentists and physicians. This led to the advent of OMFS. This is strictly a medical specialty in France, and surgeons with a lack of dental training can exceed their competence in dealing with some types of oral and maxillofacial pathology.

Dental education is essential for understanding the jaws and the face. Medical education is essential for understanding the human body. In order to practice the full scope of oral and maxillofacial surgery, the trainee must complete a curriculum that includes dental, medical, and surgical components. The question has now become whether the ideal curriculum is a formal dental degree with additional medical education, a formal medical degree with additional dental education, or a dual degree. For each trainee, years of residency and possibly a fellowship must be added. This leads to a lengthy training period - far longer than other surgical specialties. Most countries are now moving to dual degree trainingin OMFS, while keeping their OS specialty. Programs that shorten undergraduate medical and dental studies are being considered, but they are controversial since they may dilute important areas of study. We must continue to work to strengthen the specialty and to prevent the training period from becoming excessive.

\section{We have to move ahead.}

